\title{
Le calcul des courants de Foucault, en trois dimensions, en présence de corps à haute perméabilité magnétique
}

\author{
A. Bossavit \\ Electricité de France, Etudes et Recherches, 1 avenue du Général de Gaulle, 92141 Clamart, France
}

(Reçu le 29 mai 1987, révisé le 2 février 1988, accepté le 26 février 1988)

\begin{abstract}
Résumé. - Après un rappel des principes de la méthode des éléments finis en calcul des champs, on décrit une méthode de calcul des courants de Foucault en trois dimensions basée sur l'emploi d'éléments finis particuliers (associés aux arêtes du maillage et non aux nœuds). On étend la méthode à une classe assez générale de configurations, caractérisée par la présence possible de corps à haute perméabilité. On montre comment éviter de mailler ceux-ci et on étudie la notion de potentiels magnétiques flottants qui apparaît alors.
\end{abstract}

\begin{abstract}
After a recall on finite element techniques, a method is described for the computation of eddycurrents in three dimensions. This method, which is characterized by the use of unconventional (edge-based) finite elements, is further extended to a fairly large class of configurations, characterized by the presence of highly permeable bodies. Only the boundaries of such bodies need to be meshed : this is shown in the paper, and the notion of floating magnetic potentials which emerges in the process is pointed out.
\end{abstract}

\section{Introduction.}

Le calcul numérique des champs magnétiques, par des méthodes d'approximation du type éléments finis, est maintenant chose courante tant dans la recherche en électrotechnique que chez certains constructeurs, mais le plus souvent sur des configurations bidimensionnelles (présence d'un axe de symétrie, ou d'une direction d'invariance du système étudié). Le calcul en trois dimensions, s'il atteint le stade des applications industrielles dans le cas de la magnétostatique $[10,13,15]$, reste encore largement un objectif de recherche dans le cas des courants de Foucault. Le but du présent article est de décrire une méthode de calcul appropriée, en donnant les informations nécessaires à sa mise en œuvre par un programmeur expérimenté.

Ce faisant, nous souhaitons contribuer à la diffusion d'un certain savoir-faire dans ce domaine, acquis au cours du développement du code de calcul Trifou $[2,7,16]$ aux Etudes et Recherches de EDF. Ce code, qui permet de calculer des champs magnétiques non permanents dans une classe assez générale de configurations tridimensionnelles, est fondé sur une technique d'éléments finis particulière, où les inconnues sont non pas les valeurs du champ aux nœuds d'un maillage, mais les circulations le long des arêtes. Il est le résultat d'un effort engagé à partir de 1978 pour étudier, au début le chauffage par induction, plus tard des méthodes de contrôle non destructif par courants de Foucault. D'autres applications sont venues depuis [16].

Toutefois, cet article n'est pas une description analytique de l'outil de calcul que constitue Trifou, et encore moins un manuel de référence. C'est un exposé des idées à l'œuvre dans sa conception, étant entendu que l'importance relative de ces différentes idées ne nous est souvent apparue qu'après coup.

La plupart de ces idées peuvent être indiquées à propos du cas particulier suivant (Fig. 1). Considérons une bobine inductrice $S$ (la source du champ) et un conducteur $C$. Connaissant le courant (variable) dans $S$, comment calculer le champ magnétique dans tout l'espace (noté $E$ ) et les courants induits dans C?

Ce problème se divise en deux sous-problèmes : si l'on connaît la composante tangentielle du champ magnétique sur la frontière $\partial C$ du conducteur $C$, on peut (grâce à la méthode des éléments finis) calculer champ et courant à l'intérieur de $C$ et en déduire l'autre composante (normale) du champ, ou de façon équivalente, la composante tangentielle du champ électrique. On a ainsi une sorte d'impédance du conducteur vue du bord. Le deuxième sousproblème consiste à procéder de même pour le 


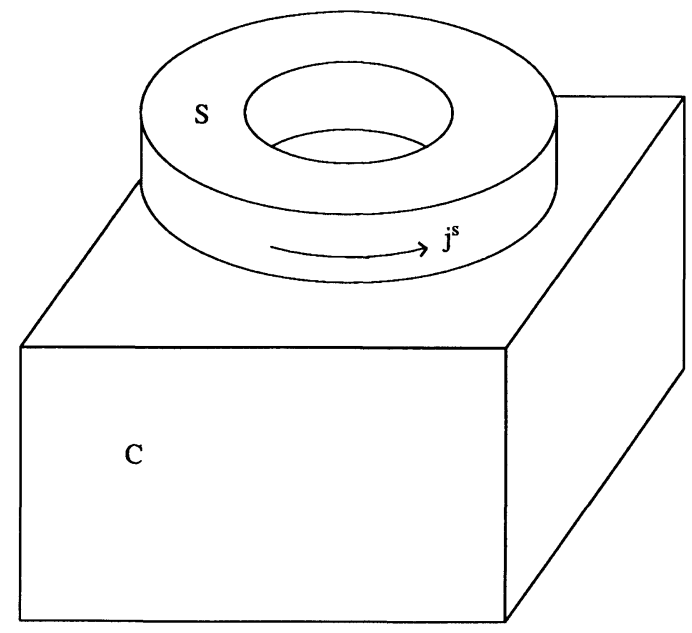

Fig. 1. - Le plus simple de tous les problèmes de courants de Foucault : connaissant la densité de courant $j^{s}$ dans $S$, calculer les courants induits dans le conducteur C.

[The simplest of eddy-current problems: Given $j^{s}$, the current density in $S$, compute the eddy-currents in the conductor $C$.]

champ extérieur. Il se ramène à la résolution d'une équation intégrale sur $\partial C$ et donc n'exige pas de maillage de l'extérieur du conducteur. Reste à ajuster les deux impédances, ce qui fournit la valeur, jusque-là inconnue, du champ tangentiel.

Sur ce cas, nous mettons en évidence trois idées clés : 1) la possibilité, grâce au découplage en deux sous-problèmes qu'on vient d'esquisser, de ne pas utiliser la méthode des éléments finis en dehors du conducteur (bien que le champ extérieur ne soit pas nul et doive être calculé), 2) l'intérêt des éléments (finis) d'arête, utilisés pour le calcul du champ intérieur, qui sont la principale particularité de Trifou, 3) l'importance, pour bien articuler tous ces outils, d'une formulation variationnelle des équations du champ:

On sait en effet que les méthodes numériques d'approximation des champs (de toute nature: champs de température, de déplacements, de vitesses...) sont plus faciles à établir et à analyser dans un cadre variationnel. L'expression de la loi fondamentale de la dynamique par le principe des travaux virtuels, par opposition à la relation $F=m \gamma$, est l'exemple le plus connu d'une telle approche variationnelle. Ces méthodes variationnelles sont aujourd'hui établies en Mécanique, en particulier en calcul des structures, mais pas encore au même degré en Electricité, et il nous a paru nécessaire d'en rappeler d'abord les principes, à propos du cas particulier de la magnétostatique.

Au terme de la première partie de cet article, on aura donc tous les éléments pour résoudre numériquement le problème de la figure 1 . Mais il est bien clair que les configurations d'intérêt pratique sont plus complexes. En particulier, les dispositifs de chauffage par induction, ou de contrôle non destructif, les transformateurs, les machines, etc., comportent toujours des parties ferromagnétiques, à perméabilité élevée, destinées à canaliser, ou à confiner, le champ magnétique. Or, lorsque la perméabilité est très élevée dans une région de l'espace, le champ magnétique y est très petit. (Ceci, en règle générale ; il y a des exceptions, pour lesquelles nous renvoyons à [6].) Donc, il n'y a pas lieu de rechercher une approximation du champ, dans une telle région, par des méthodes numériques, et on souhaite éviter ce calcul, pour deux raisons. Premièrement, le souci d'économiser le temps de calcul correspondant à la résolution d'un système d'équations approchées inutilement grand, puisqu'on sait par avance que certaines inconnues sont nulles, ou quasi-nulles. Deuxièmement, celui d'éviter les difficultés numériques inhérentes à tout calcul où figure un petit paramètre, ici le rapport $\varepsilon=\mu_{0} / \mu$ de la perméabilité du vide à celle des culasses ferromagnétiques. (On qualifiera désormais de magnétiques, par abus de langage, ces régions où $\varepsilon$ est petit.)

La deuxième partie est consacrée à ce problème. La figure 2 (qui sera commentée plus longuement au début de la deuxième partie) peut donner une idée des configurations que l'on rencontre en pratique. On verra que les idées de la première partie restent valables, mais que des difficultés techniques viennent compliquer l'implémentation.

Par la suite, on fera souvent des hypothèses simplificatrices telles que $\mu=\mu_{0}$ partout, matériaux homogènes, etc. Ce sera seulement pour la clarté, et non à cause de limitations intrinsèques de la méthode. Par exemple, on supposera $S$ et $C$ distincts. En fait, la source du champ pourrait être une densité de courant connue dans une partie du conducteur (ne constituant pas forcément un circuit fermé). La source pourrait aussi (comme dans [2] et [3]) être une ou des forces électromotrices imposées. Aucune de ces variantes ne présente de difficulté supplémentaire sérieuse.

Une hypothèse restrictive d'importance, toutefois, est que champs et courants sont sinusoïdaux. De ce fait, toutes les entités $h, b, j$, etc., sont à valeurs complexes, ce qui exclut les lois de comportement ( $b$ en fonction de $h$ ) non linéaires. On a aussi écarté de cet exposé les difficultés de nature topologique dues à la présence éventuelle de boucles de courants (potentiels magnétiques multivoques) [7]. Cette éventualité est prévue dans Trifou, mais nous renvoyons à [17] pour le détail de son traitement.

Le plan de l'article est le suivant :

Première partie (sans corps à haute perméabilité)

1. Exposé du problème

2. Le calcul du champ intérieur au conducteur 


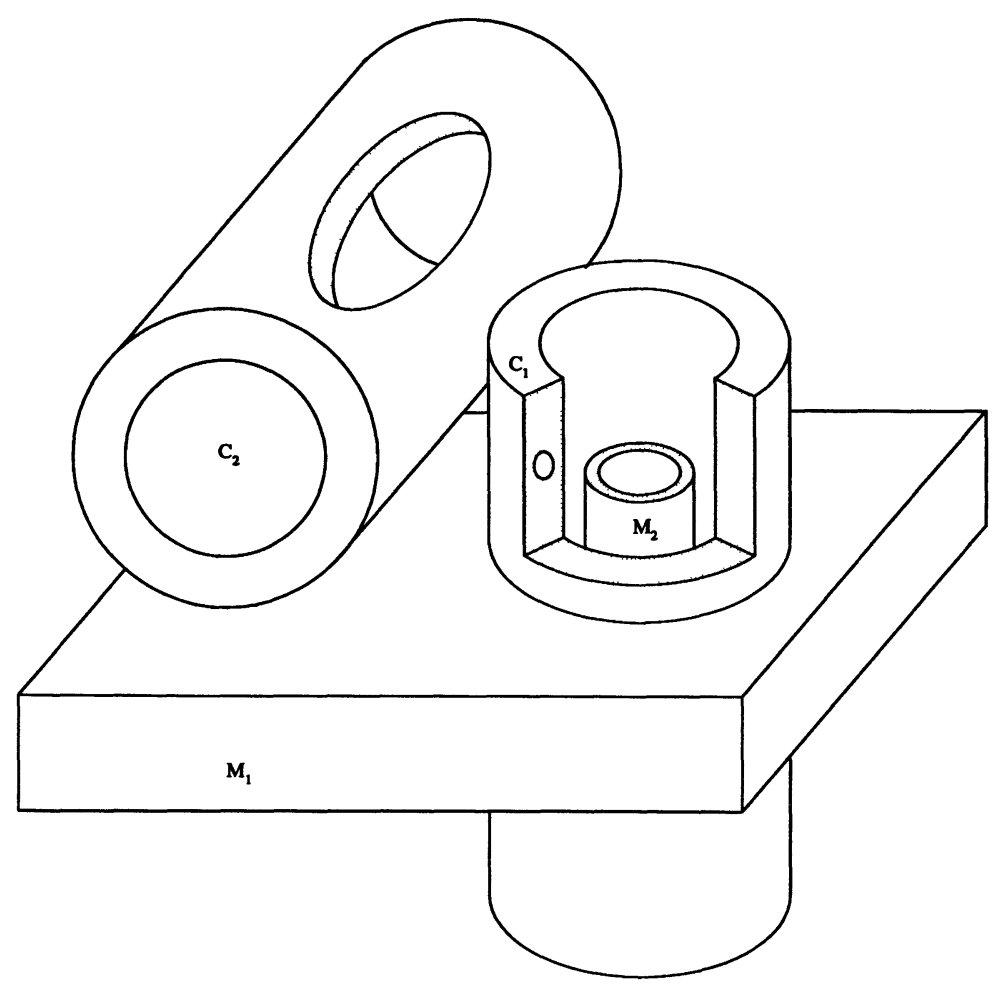

Fig. 2. - Configuration imaginaire, mais proche de celles rencontrées en contrôle non destructif. $C_{1}$ et $C_{2}$ sont des tuyaux conducteurs ; $M_{1}$ (la plaque support) et $M_{2}$ (le noyau de la bobine de contrôle) sont ferromagnétiques à haute perméabilité. (Les parties grisées sont des coupes, destinées à montrer l'intérieur des pièces.)

[An imaginary configuration, but typical of applications to non-destructive testing. $C_{1}$ and $C_{2}$ are electically conducting pipes ; $M_{1}$ (the supporting slab) and $M_{2}$ (the core of the probe) are ferromagnetic, with a high permeability. (Dotted parts are cuts, in order to show the inside.)]

2.1 Cas de la magnétostatique

2.2 La méthode de Galerkine

2.3 Cas des courants de Foucault

2.4 Remarques

3. Calcul du champ dans tout l'espace

3.1 Formulation variationnelle

3.2 Discrétisation

Conclusion

Deuxième partie (avec corps à haute perméabilité)

1. Exposé du problème

2. Formulation

3. Discrétisation

Conclusion

\section{Conclusion générale}

Bibliographie

\section{Première partie}

\section{Exposé du problème.}

On considère dans cette partie le problème de la figure 1 , dont la définition formelle est la suivante.
1) Sont données deux régions de l'espace, $S$ et $C$, bornées, disjointes, dont les frontières sont les surfaces $\partial S$ et $\partial C$. L'espace entier est noté $E$.

2) La région $S$ (source du champ) porte une densité de courant de la forme $\operatorname{Re}\left[j^{s} \exp (i \omega t)\right]$ où $j^{s}$ est une fonction complexe $x \rightarrow j^{s}(x)$ de la position. On suppose $j^{s}$ prolongé par 0 à l'extérieur de $S$, et $\operatorname{div} j^{s}=0$ dans $E$.

3) La région $C$ (le conducteur) est caractérisée par une résistivité $\rho$, fonction de la position $x$, $\rho(x)>0$.

4) $C$ est en un seul morceau (« connexe»), ainsi que sa frontière. De plus $C$ est simplement connexe (pas de boucles).

5) La perméabilité $\mu$ est fonction de la position $x$, avec $\mu(x) \geqslant \mu_{0}$ partout, et $\mu(x)=\mu_{0}$ dans $E-C$.

A l'occasion, on pourra simplifier l'exposé en supposant $\rho$ et $\mu$ indépendants de $x$, mais ce n'est pas une restriction nécessaire.

Dans ces conditions, calculer les courants induits dans $C$ et le champ $h$ partout. 
Les équations de la situation, bien connues, sont :

$$
\begin{gathered}
i \omega b+\operatorname{rot} e=0 \quad \text { (loi de Faraday), } \\
b=\mu_{0} h, \\
\operatorname{rot} h=j \quad \begin{array}{l}
\text { (théorème d'Ampère) }, \\
j=j^{s}+\sigma e,
\end{array}
\end{gathered}
$$

où $\sigma$ (la conductivité) vaut $1 / \rho$ dans $C$ et 0 ailleurs (en particulier dans $S$ ). Remarquer que

$$
\operatorname{div} b=0
$$

est conséquence de (1).

Conformément à la stratégie de découplage esquissée dans l'introduction, on suppose connue (provisoirement) la composante tangentielle du champ $h$ sur $\partial C$, que l'on appellera $g$, et on traite d'abord le problème intérieur (calculer $h$ et $j$ dans $C$ ), puis le problème extérieur.

\section{Calcul du champ intérieur au conducteur.}

2.1 CAS DE LA MAGNÉTOSTATIQUE. - Envisageons d'abord le problème magnétostatique dans le conducteur, à seule fin de décrire simplement la méthode variationnelle et le principe de la méthode des éléments finis. Le lecteur au fait de ces idées peut sauter cette section, qui est indépendante du reste.

Les équations sont maintenant (pas de courant induit dans $C$ )

$$
\operatorname{rot} h=0, \quad b=\mu_{0} h, \quad \operatorname{div} b=0
$$

dans $C$. Puisque $C$ est simplement connexe, il existe (à une constante additive près) un potentiel magnétique $\varphi$ dans $C$, tel que $h=-\operatorname{grad} \varphi$. Appelons $\varphi^{s}$ (la source du champ) le potentiel magnétique, supposé connu, sur $\partial C$. Alors, $\varphi=\varphi^{s}$ sur $\partial C$. La méthode variationnelle est souvent décrite comme la recherche, dans une classe de potentiels a priori possibles, celui qui correspond au minimum de l'énergie du système. Ceci (qui est parfaitement correct dans le cas présent) est un peu restrictif, et on préférera la description qui suit.

Des trois équations (6), celle du milieu décrit le comportement du matériau. Les deux autres, symétriques, décrivent les lois de l'électromagnétisme. Choisissons l'une de ces lois, par exemple rot $h=0$ (on pourrait prendre l'autre), et cherchons à décrire tous les champs qui la satisfont. Ils sont de la forme $h=-\operatorname{grad} \varphi$, où $\varphi$ est une fonction $\operatorname{sur} C$ pourvue de certaines propriétés mathématiques : assez régulière pour que prendre son gradient ait un sens, et telle que l'énergie magnétique associée soit finie. Sans insister sur la définition de cette classe de fonctions (c'est un des espaces de Sobolev [14]) appelons-la $\Phi$. Si $\varphi$ est le potentiel magnétique cherché, on sait d'avance que $\varphi \in \Phi$.
Soit maintenant

$$
\Phi^{s}=\left\{\varphi \in \Phi: \varphi=\varphi^{s} \text { sur } \partial C\right\},
$$

qui sont les fonctions de $\Phi$ qui satisfont à la condition imposée au potentiel sur la surface. De nouveau, $\Phi^{s}$ contient le potentiel cherché. En fait, chercher $\varphi$ dans $\Phi^{s}$ revient à se conformer aux conditions aux limites imposées et à l'équation rot $h=0$. Restent $\operatorname{div} b=0$ et la loi $b=\mu_{0} h$.

Ici intervient la notion de formulation faible. Grâce à la formule de Green suivante :

$$
\int_{C} b \cdot \operatorname{grad} \varphi=-\int_{C} \operatorname{div} b \varphi+\int_{\partial C} n \cdot b \varphi,
$$

facile à vérifier en intégrant par parties (on note $n$ le champ des vecteurs normaux, de longueur 1 , sur $\partial C$, sortants par rapport à $C$ ), on voit que $\operatorname{div} b=0$ s'écrit aussi

$$
\int_{C} b \cdot \operatorname{grad} \varphi^{\prime}=0 \quad \forall \varphi^{\prime} \in \Phi^{0}
$$

où $\Phi^{0}$ est la classe des potentiels nuls sur $\partial C$, soit

$$
\Phi^{0}=\{\varphi \in \Phi: \varphi=0 \text { sur } \partial C\} .
$$

L'avantage de la formulation faible (8) sur la formulation forte (5) apparaîtra au moment de la discrétisation par éléments finis.

Le dernier pas consiste à tenir compte de la loi de comportement $b=\mu_{0} h, \quad$ donc à écrire $b=-\mu_{0} \operatorname{grad} \varphi$, et à porter dans (8). Les équations (6), plus les conditions aux limites, s'expriment alors sous la forme suivante :

$$
\left\{\begin{array}{l}
\operatorname{chercher} \varphi \in \Phi^{s} \text { tel que } \\
\int_{C} \mu \operatorname{grad} \varphi \cdot \operatorname{grad} \varphi^{\prime}=0 \quad \forall \varphi \in \Phi^{0} .
\end{array}\right.
$$

C'est la formulation variationnelle annoncée. Si l'on introduit la quantité connue sous le nom de coénergie magnétique, soit

$$
W(\varphi)=1 / 2 \int_{C} \mu|\operatorname{grad} \varphi|^{2}
$$

on peut vérifier, en dérivant par rapport à $\varphi$ dans (10), que (9) est équivalent à $\varphi \in \Phi^{s}$ et

$$
W(\varphi) \leqslant W\left(\varphi^{\prime}\right) \quad \forall \varphi^{\prime} \in \Phi^{s},
$$

autrement dit, le potentiel cherché est celui de coénergie minimale.

On peut considérer (9) comme un système linéaire d'une infinité d'équations à une infinité d'inconnues. Le pas suivant dans la démarche consiste à se ramener, au prix d'une erreur d'approximation (inévitable, mais contrôlable), à un nombre fini d'équations et d'inconnues (méthode de Galerkine). Le système peut alors être résolu. 
2.2 LA MÉTHOdE DE GALERKINE. - Soit $\mathfrak{C}$ un pavage simplicial de $C$, c'est-à-dire un ensemble de tétraèdres, dont la réunion est $C \cup \partial C$, et qui n'ont en commun deux à deux qu'un triangle, une arête, un sommet, ou rien. (Il est permis de prendre des images de tétraèdres par des applications continues, de manière à avoir des surfaces d'éléments qui s'adaptent à la courbure de $\partial C$.) On fera usage des notations suivantes:

$\mathcal{C}$ : ensemble de tous les tétraèdres;

$\mathcal{S}$ : ensemble de tous les sommets;

$\mathrm{S}_{0}$ : sommets intérieurs à $C$.

Ainsi, $\mathcal{S}-\mathcal{S}_{0}$ est l'ensemble des sommets frontaliers.

A chaque sommet $s \in \mathcal{S}$, faisons correspondre la fonction barycentrique $\lambda_{s}$ ainsi définie : dans tout tétraèdre dont $s$ est un sommet, $\lambda_{s}(x)$ est la coordonnée barycentrique de $x$ relativement au sommet $s$. Partout ailleurs, $\lambda_{s}$ est nulle. (Rappelons que tout point $x$ d'un tétraèdre de sommets $x_{i}$ s'écrit de façon unique $x=\sum \lambda_{i} x_{i}$, c'est-à-dire comme barycentre

des sommets, avec comme poids les $\lambda_{i}$, qui sont les coordonnées barycentriques de $x$.)

Maintenant, soit $U$ l'ensemble de tous les vecteurs $u=\left\{u_{\sigma}: \sigma \in \mathcal{S}\right\}$ dont les coordonnées sont des valeurs (réelles ou complexes) associées aux sommets du pavage. On voit qu'à tout $u \in U$ correspond une fonction

$$
\varphi=\sum_{\sigma \in \delta} u_{\sigma} \lambda_{\sigma}
$$

qui par construction appartient à l'espace $\Phi$. Soit alors $\Phi_{\mathcal{C}}$ la classe de toutes les fonctions $\varphi$ de la forme (12) pour toutes les valeurs possibles de $u$. On voit que $\Phi_{\tau}$ est un sous-espace vectoriel de $\Phi$, dont la dimension est le nombre de sommets de $\delta$, et qu'il y a correspondance biunivoque entre $U$ et $\Phi_{\mathcal{\zeta}}$.

Par analogie, soit

$$
\begin{aligned}
& U^{s}=\left\{u \in U: u_{\sigma}=\varphi^{s}(\sigma) \text { si } \quad \sigma \notin S_{0}\right\}, \\
& U^{0}=\left\{u \in U: u_{\sigma}=0 \quad \text { si } \quad \sigma \notin \mathcal{S}_{0}\right\} \text {, }
\end{aligned}
$$

et, pour les espaces de fonctions correspondants,

$$
\begin{aligned}
& \Phi_{\mathcal{G}}^{s}=\left\{\sum_{\sigma \in \delta} u_{\sigma} \lambda_{\sigma}: u \in U^{s}\right\}, \\
& \Phi_{\mathcal{C}}^{0}=\left\{\sum_{\sigma \in \delta} u_{\sigma} \lambda_{\sigma}: u \in U^{0}\right\},
\end{aligned}
$$

Reprenons la recherche du potentiel, toujours sous la forme (12), mais avec $u \in U^{s}$. Les valeurs de $u_{\sigma}$ étant connues pour $\sigma \in \partial C$, le nombre d'inconnues est celui des sommets de $\boldsymbol{S}_{0}$. On appelle degrés de liberté de $\varphi$ ces inconnues.

On cherche donc $\varphi$ dans $\Phi_{\mathscr{b}}^{s}$, qui est contenu dans $\Phi^{s}$, et plus petit. Sauf accident heureux, on ne peut donc plus espérer trouver le vrai $\varphi$, mais seulement une approximation, d'autant meilleure que les fonctions de $\Phi^{s}$ sont bien approchées par celles de $\Phi_{\zeta}^{s}$. (Quantifier cette notion de qualité d'approximation, en particulier montrer comment elle s'améliore avec la finesse du maillage, est du ressort de l'Analyse Numérique.) On notera que si $h=-\operatorname{grad} \varphi, \operatorname{avec} \varphi \in \Phi_{\mathcal{C}}^{s}$, on a bien rot $h=0$. Mais si l'on pose $b=\mu h=-\mu \operatorname{grad} \varphi$, l'équation $\operatorname{div} b=0$ ne peut pas être satisfaite. En effet, par construction des $\varphi$ de $\Phi^{s}$, leur gradient n'est pas continu au passage des facettes du maillage. Seule est continue la composante tangentielle de ce champ, ce qui du reste est tout ce que la physique requiert de ce champ. Mais on doit renoncer à la continuité normale de $b$.

Donc la forme forte, $\operatorname{div} b=0$, de l'équation sur $b$, ne peut être satisfaite exactement. En revanche, la forme faible (8) est recevable, à condition de remplacer $\Phi^{0}$ par $\Phi_{\zeta}^{0}$ : en effet, de cette façon, on n'impose à $b$, donc à $h$, qu'un nombre fini de contraintes (autant que de sommets dans $S_{0}$ ).

En fin de compte, la méthode de Galerkine consiste donc à résoudre le problème (9), mais en rajoutant l'indice $\mathfrak{C}$ là où il faut :

$$
\left\{\begin{array}{l}
\operatorname{chercher} \varphi \in \Phi_{\mathscr{C}}^{s} \text { tel que } \\
\int_{C} \mu \operatorname{grad} \varphi \cdot \operatorname{grad} \varphi^{\prime}=0 \quad \forall \varphi^{\prime} \in \Phi_{\mathscr{C}}^{0} .
\end{array}\right.
$$

Si l'on pose, pour deux sommets $\sigma$ et $s$ de $\mathcal{S}$,

$$
A_{\sigma s}=\int_{C} \mu \operatorname{grad} \lambda_{\sigma} \cdot \operatorname{grad} \lambda_{s},
$$

d'où une matrice carrée $A$, on voit, d'après la correspondance établie plus haut, que (13) équivaut au problème suivant :

$\left\{\begin{array}{l}\text { chercher } \varphi \in U^{s} \text { tel que } \\ \sum_{\sigma \in \delta, s \in \delta_{0}} A_{\sigma s} u_{\sigma} u_{s}^{\prime}=0 \quad \forall u^{\prime} \in U^{0} .\end{array}\right.$

Il s'agit bien là d'un système linéaire, de dimension égale au nombre de sommets de $\boldsymbol{S}_{0}$, que l'on peut résoudre avec un ordinateur. Il s'écrit aussi, plus simplement,

$$
\sum_{\sigma \in \delta} A_{\sigma s} u_{\sigma}=0 \quad \forall s \in \mathcal{S}_{0} .
$$

On peut remarquer que résoudre ce système revient à résoudre le problème de minimum (11), mais dans $\Phi_{\mathfrak{G}}^{s}$ au lieu de $\Phi^{s}$, ce qui donne une valeur par excès de la coénergie.

Les idées exposées ici à propos de la magnétostatique seront reprises dans toute la suite, mais sous d'autres formes.

2.3 CAS DES Courants de Foucault. - Revenons maintenant au calcul des courants de Foucault, 
connaissant la partie tangentielle de $h$, soit $g^{s}$, sur $\partial C$. Comme rot $h=j$ n'est plus nul, on ne peut plus se servir d'un potentiel scalaire. On retient toutefois l'idée de chercher $h$ parmi une certaine classe de champs : ceux de carré intégrable dans $C$ (c'est-àdire de coénergie finie), à rotationnel de carré intégrable (de sorte que la puissance Joule associée soit finie). Le nom technique de cet espace de champs est $\mathbb{L}_{\text {rot }}^{2}(C)$. On pose donc $H=\mathbb{L}_{\text {rot }}^{2}(C)$, puis

$$
H^{s}=\left\{h \in H: n \wedge h=n \wedge g^{s} \text { sur } \partial C\right\}
$$

(de sorte que la partie tangentielle de $h$ soit égale à $\left.g^{s}\right)$, et

$$
H^{0}=\{h \in H: n \wedge h=0 \text { sur } \partial C\} .
$$

On demandera à $h$ de satisfaire la loi de Faraday (1) sous la forme faible suivante :

$i \omega \int_{C} b \cdot h^{\prime}+\int_{C} e \cdot \operatorname{rot} h^{\prime}=0 \quad \forall h^{\prime} \in H^{0}$

(remarquer l'analogie avec (8)). Reste à tenir compte des lois de comportement $b=\mu h$ et $e=\rho j=\rho$ rot $h$, en les portant dans (15). On obtient (comparer avec (9)) :

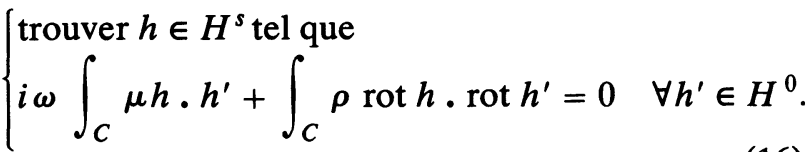

Telle est la forme variationnelle du problème des courants de Foucault à l'intérieur du conducteur. (On remarquera que (16) n'est pas équivalent à un problème de minimum, cette fois.)

Pour discrétiser (16), on recourt de nouveau à un pavage $\mathcal{C}$ de $C$ par des tétraèdres. Aux notations introduites plus haut, rajoutons

$\mathcal{A}$ : ensemble de toutes les arêtes du maillage, $\mathcal{A}_{0}$ : arêtes internes à $C$.

A chaque arête $a$ de $\mathcal{A}$, caractérisée par deux sommets $s$ et $\sigma$, associons le champ de vecteurs

$$
w_{a}=\lambda_{\sigma} \operatorname{grad} \lambda_{s}-\lambda_{s} \operatorname{grad} \lambda_{\sigma} .
$$

Il est nul partout, sauf sur les tétraèdres (ou triangles) qui ont en commun l'arête $a$. Sa partie tangentielle est continue au passage des facettes du maillage. Par construction, la circulation de $w_{a}$ sur l'arête $a$ (orientée de $\sigma$ vers $s$ ) est égale à 1 , et elle est nulle sur toutes les autres arêtes. Nous allons utiliser les $w_{a}$ comme éléments finis, donc ces circulations apparaîtront comme les degrés de liberté relatifs à ces éléments. Ceci les distingue des éléments finis courants, dont les degrés de liberté sont liés aux nœuds du maillage.

La circulation d'un champ $h$ sur une arête $a$ sera notée $\int_{a} t \cdot h$, où $t$ est un champ de vecteurs tangents à $a$ de longueur 1 , orientés dans le sens de a.

Soit à nouveau $U$ l'ensemble de tous les vecteurs $u=\left\{u_{a}: a \in \mathcal{A}\right\}$, dont les composantes sont associées maintenant aux arêtes du maillage, et non plus aux sommets. Soit

$$
\begin{aligned}
& U^{s}=\left\{u \in U: u_{a}=\int_{a} t \cdot g^{s} \quad \text { si } \quad a \notin \mathcal{A}_{0}\right\}, \\
& U^{0}=\left\{u \in U: u_{a}=0 \quad \text { si } \quad a \notin \mathcal{A}_{0}\right\} .
\end{aligned}
$$

A ces espaces vectoriels de dimension finie correspondent, de façon biunivoque, les sous-espaces suivants de $H$ :

$$
\begin{aligned}
& H_{\mathcal{C}}^{s}=\left\{\sum_{a \in \mathcal{A}} u_{a} w_{a}: u \in U^{s}\right\}, \\
& H_{\mathfrak{C}}^{0}=\left\{\sum_{a \in \mathcal{A}} u_{a} w_{a}: u \in U^{0}\right\} .
\end{aligned}
$$

La méthode de Galerkine, à nouveau, consiste à chercher $h$ dans $H_{\mathfrak{b}}^{s}$, c'est-à-dire à déterminer autant de degrés de liberté inconnus qu'il y a d'arêtes internes. Pour cela, il faut imposer exactement autant de contraintes, donc remplacer $H^{0}$ par $H_{\mathfrak{\zeta}}^{0}$ dans (16). On débouche donc sur le problème discrétisé suivant :

$$
\left\{\begin{array}{l}
\text { trouver } h \in H_{\mathcal{C}}^{s} \text { tel que } \\
i \omega \int_{C} \mu h \cdot h^{\prime}+\int_{C} \rho \operatorname{rot} h . \operatorname{rot} h^{\prime}=0 \forall h^{\prime} \in H_{\mathcal{C}}^{0} .
\end{array}\right.
$$

On voit que (18) équivaut au système linéaire suivant :

$$
\left\{\begin{array}{l}
\text { trouver } u \in U^{s} \text { tel que } \\
\sum_{a \in A, \alpha \in A_{0}} A_{a \alpha} u_{a} u_{\alpha}^{\prime}=0 \quad \forall u^{\prime} \in U^{0}
\end{array}\right.
$$

c'est-à-dire, plus simplement,

$$
\sum_{a \in \mathcal{A}} A_{a \alpha} u_{a}=0 \quad \forall \alpha \in \mathcal{A}_{0}
$$

avec cette fois

$$
A_{a \alpha}=i \omega \int_{C} \mu w_{a} \cdot w_{\alpha}+\int_{C} \rho \operatorname{rot} w_{a} \cdot \operatorname{rot} w_{\alpha} .
$$

Le nombre d'inconnues est celui des arêtes internes.

2.4 REMARQUES. - Ainsi, nous avons reproduit strictement la démarche qui réussit pour la magnétostatique. La raison pour laquelle l'opérateur rot, dans (15), est passé devant $h^{\prime}$ alors qu'il est devant $e$ dans l'expression forte de la loi de Faraday, est la même pour laquelle (8) a été substitué à div $b=0$ plus haut : par construction de $h$, son rotationnel existe, donc on peut écrire $j=\operatorname{rot} h$, si $h \in H_{\mathfrak{Z}}^{s}$. Mais 
si la composante normale de ce champ $j$ est continue au passage des facettes, puisque $\operatorname{div} j=0$, la composante tangentielle ne l'est pas. Il en va de même de $e$, puisque $e=\rho j$, donc on ne peut pas prendre la rotationnelle de $e$ (qui serait, mathématiquement parlant, une distribution). La loi de Faraday n'est donc satisfaite (pour la solution approchée) qu'au sens faible, en moyenne en quelque sorte. A fortiori, $\operatorname{div} b=0$ n'est valable qu'au sens faible alors que rot $h=j$ vaut au sens fort. Ce phénomène (on ne peut gagner sur les deux tableaux) est général dans la méthode des éléments finis : l'approximation obtenue ne peut jamais satisfaire aux deux exigences de la physique (continuité tangentielle de $h$, continuité normale de $b$ ) en même temps. On ne peut que choisir celle à laquelle on tient (ici, la première) et se contenter d'avoir l'autre au sens faible.

On a longtemps cherché à résoudre le problème des courants de Foucault à l'aide d'éléments finis traditionnels (ceux utilisés plus haut pour le potentiel), mais avec trois degrés de liberté pour $h$ à chaque sommet du maillage, un par composante. De la sorte, les trois composantes de $h$ sont continues. Ceci, loin d'être un avantage, impose au niveau des interfaces matérielles (où $\mu$ ou $\rho$ changent de valeur) une continuité dont on ne veut pas, et de toute façon, même dans le cas d'un matériau homogène, ces méthodes ne convergent pas [1]. Une autre méthode très répandue (et celle-là, correcte), dite « $T-\Omega$ » [9], consiste à corriger cet excès de continuité en ajoutant au champ $h$ précédent (noté $T$ dans [9] et depuis) le gradient d'un potentiel $\Omega$, lui-même approché par des éléments nodaux. On peut enfin travailler avec le potentiel vecteur, approché soit par des éléments nodaux, soit par des éléments d'arêtes. Bien qu'il n'y ait pas cette fois d'objection théorique à l'emploi d'éléments nodaux, il semble [1] que les éléments d'arête soient plus efficaces en termes de temps de calcul. On a montré dans [5] que cette dernière méthode (potentiel vecteur et éléments d'arête) est duale de celle exposée ici, dans la mesure où elle assure $\operatorname{div} b=0$ $\mathrm{au}$ sens fort et le théorème d'Ampère seulement au sens faible. Les éléments finis d'arête ont été décrits dans [12] mais pas sous la forme (17), inspirée de [18]. Celle-ci a l'avantage de donner directement une base de $H_{\zeta}$, ce qui facilite l'assemblage des éléments.

\section{Calcul du champ dans tout l'espace.}

Passons au problème complet, tenant compte du champ extérieur. Cette fois, la source du champ n'est plus $g^{s}$ (le champ tangentiel sur $\partial C$ ) mais le courant $j^{s}$ dans $S$.

Définissons d'abord un champ-source $h^{s}$, qui n'est pas le champ physique réel, mais va servir à le déterminer.
On pose, pour tout $x$ de $E$ :

$$
a^{s}(x)=\frac{1}{4 \pi} \int_{s} \frac{j^{s}(y)}{|x-y|} d y
$$

(où $|x-y|$ est la distance des deux points $x$ et $y$, et dy l'élément de volume). Puis on définit

$$
h^{s}=\operatorname{rot} a^{s}
$$

dans $E$ tout entier. Comme, par hypothèse,

$$
\operatorname{div} j^{s}=0
$$

on a

$$
\operatorname{rot} h^{s}=j^{s}, \operatorname{div} h^{s}=0
$$

3.1 Formulation VARiationNelle. - Soit $H$ l'espace (connu sous le nom de $\mathbb{L}_{\text {rot }}^{2}(E)$ ) des champs définis sur tout l'espace, d'énergie finie, et ayant un rotationnel de carré intégrable. On pose

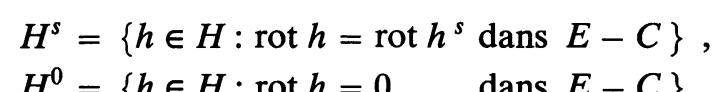

Noter que $h^{s}$ est dans $H^{s}$. La formulation variationnelle du problème est

$\left\{\begin{array}{l}\text { trouver } h \in H^{s} \text { tel que } \\ i \omega \int_{E} \mu h \cdot h^{\prime}+\int_{C}^{\rho} \rho \operatorname{rot} h \cdot \operatorname{rot} h^{\prime}=0 \quad \forall h^{\prime} \in H^{0} .\end{array}\right.$

On démontre que (22) a une solution unique, et il est facile de voir qu'elle vérifie le théorème d'Ampère (par construction) et la loi de Faraday (voir [4] pour une preuve).

Le problème est qu'on ne peut pas maintenant définir directement des ensembles de champs analogues aux $H_{\mathfrak{C}}$ utilisés plus haut : il faudrait mailler tout l'espace. La comparaison entre (16) et (22) suggère de réécrire ainsi l'équation (22) :

$$
\begin{aligned}
i \omega \int_{E-C} & \mu h \cdot h^{\prime}+i \omega \int_{C} \mu h \cdot h^{\prime}+ \\
& +\int_{C} \rho \operatorname{rot} h \cdot \operatorname{rot} h^{\prime}=0 \quad \forall h^{\prime} \in H^{0} .
\end{aligned}
$$

ce qui désigne le problème à résoudre : comment évaluer l'intégrale sur $E-C$ de (23) sans pour autant mailler l'espace extérieur $E-C$, donc uniquement en fonction d'informations données sur la frontière $\partial C$ ?

Ici intervient le découplage des problèmes extérieur et intérieur. Supposons connu le champ tangentiel sur $\partial C$. Comme $h$ dans $E-C$ est de la forme $h^{s}+\operatorname{grad} \Phi$ (d'après (21)), cela revient à connaître $\Phi$ sur $\partial C$. Soit $\varphi$ ce potentiel sur $\partial C$.

Comme $\operatorname{div} h^{s}=0$ par construction, et que

$$
\operatorname{div}\left(\mu_{0}\left(h^{s}+\operatorname{grad} \Phi\right)\right)=0 \text { dans } E-C,
$$


on voit que

$$
\left\{\begin{aligned}
\Delta \Phi & =0 & & \text { dans } E-C \\
\Phi & =\varphi & & \text { sur } \partial C .
\end{aligned}\right.
$$

Appelons $R$ l'opérateur (dit de Poincaré-Steklov) qui à $\varphi$ fait correspondre $\partial \Phi / \partial n$ (c'est-à-dire $n \cdot \operatorname{grad} \Phi$, où $n$ est la normale vers l'intérieur de C).

Soit maintenant $h$ la solution, $\varphi$ son potentiel sur $\partial C$, et $h^{\prime} \in H^{0}$ quelconque. On a $h^{\prime}=\operatorname{grad} \Phi^{\prime}$ dans $E-C$. Soit $\varphi^{\prime}$ la restriction de $\Phi^{\prime}$ à $\partial C$. Alors

$$
\begin{aligned}
\int_{E-C} \mu h \cdot h^{\prime} & =\int_{E-C} \mu_{0}\left(h^{s}+\operatorname{grad} \Phi\right) \cdot \operatorname{grad} \Phi^{\prime} \\
& =\mu_{0} \int_{\partial C}\left(n \cdot h^{s}+R \varphi\right) \varphi^{\prime}
\end{aligned}
$$

grâce à la formule de Green (7) (appliquée sur $E-C$ au lieu de $C$ ), et à (24).

En portant cette expression dans (23), on obtient une nouvelle formulation variationnelle, qui porte sur des couples $\{h, \varphi\}$ (liés par le fait que $\varphi$ est un potentiel pour $h$ ), avec $h$ défini seulement sur $C$ et $\varphi$ sur $\partial C$, ce qui rend possible la discrétisation avec un maillage restreint au conducteur, toujours selon la méthode de Galerkine.

Pour cela, appelons $H \Phi$ l'espace des couples $\{h, \varphi\}$ dont le premier est la restriction à $C$ d'un élément de $H$, le second une fonction définie sur $\partial C$. Pour éviter toute confusion, on note $h_{E}$ le champ défini sur tout $E$ et $h$ ce même champ restreint au conducteur.

Soit $h_{E} \in H^{s}$. D'après (21), il existe un potentiel $\Phi$ dans $E-C$, nul à l'infini, tel que $h_{E}=h^{s}+\operatorname{grad} \Phi$ dans cette région. Soit $h$ la restriction de $h_{E}$ à $C$ et $\varphi$ celle de $\Phi$ à $\partial C$. On appalle $H \Phi^{s}$ l'espace de tous les couples $\{h, \varphi\}$ ainsi obtenus. Soit de même $H \Phi^{0}$ obtenu à partir des éléments de $H^{0}$. Le nouveau problème variationnel s'écrit

$$
\left\{\begin{array}{l}
\text { trouver }\{h, \varphi\} \in H \Phi{ }^{s} \text { tels que } \\
i \omega\left[\int_{C} \mu h \cdot h^{\prime}+\mu_{0} \int_{\partial C}\left(n \cdot h^{s}+R \varphi\right) \varphi^{\prime}\right] \\
+\int_{C} \rho \operatorname{rot} h \cdot \operatorname{rot} h^{\prime}=0 \quad \forall\left\{h^{\prime}, \varphi^{\prime}\right\} \in H \Phi^{0} .
\end{array}\right.
$$

(Il se trouve, ici, que $H \Phi^{s}$ et $H \Phi^{0}$ coïncident. En revanche, la source du champ apparaît explicitement dans l'équation, sous la forme de la composante normale $n \cdot h^{s}$.)

3.2 Discrétisation. - Pour discrétiser (26), définissons d'abord

$$
U=\left\{u_{a}: a \in \mathcal{A}_{0}, u_{s}: s \in \mathcal{S}-\mathcal{S}_{0}\right\} .
$$

Autrement dit, on a un degré de liberté par arête interne et un par sommet frontalier. Pour chaque arête frontalière $a=\{s, \sigma\}$, on pose

$$
u_{a}=u_{\sigma}-u_{s} \text {. }
$$

Soit enfin

$$
\begin{aligned}
h & =\sum_{\sigma \in \mathcal{A}} u_{a} w_{a}, \\
\varphi & =\sum_{s \in \delta-\delta_{0}} u_{s} \lambda_{s} .
\end{aligned}
$$

On vient d'obtenir un couple $\{h, \varphi\}, h$ défini sur $C, \varphi$ sur $\partial C$. Soit $H \Phi_{\succ}$ la classe de tous ces couples. Par construction, c'est-à-dire grâce à (27) et à la définition même de $w_{a}$ donnée en (17), la composante tangentielle de $h$ coüncide avec grad $\varphi$. Ce point est essentiel : il montre que les éléments finis d'arête définis en (17), que nous utilisons pour les champs de vecteurs, se marient bien avec les éléments finis nodaux utilisés pour les champs scalaires.

En fin de compte, le problème discrétisé est (26), en substituant $H \Phi_{\mathcal{C}}$ à $H \Phi^{s}$ et $H \Phi^{0}$. D'après (28) (29), il s'agit bien d'un système linéaire déterminant les degrés de liberté $u$. Il reste à calculer les coefficients de ce système (phase d'assemblage de la méthode des éléments finis). La seule chose à préciser à ce propos est le calcul de la matrice $R$ correspondant à l'opérateur $R$ qui apparaît dans (26).

En fait, ce point constitue un sujet de recherches à lui seul. On se contentera d'indiquer une méthode (qui diffère légèrement de celle utilisée dans Trifou, cf. [5]). On sait («troisième formule de Green ») que pour toute fonction $\varphi$ sur $\partial C$,

$$
\begin{aligned}
\frac{1}{2} \varphi(x)+\frac{1}{4 \pi} \int_{\partial C} & \frac{n(x) \cdot(x-y)}{|x-y|^{3}} \varphi(y) \mathrm{d} y= \\
& =\frac{1}{4 \pi} \int_{\partial C} \frac{\mathrm{d} y}{|x-y|}(R \varphi)(y)
\end{aligned}
$$

ce que l'on peut écrire, sous forme plus compacte, avec des notations évidentes,

$$
(1 / 2+H) \varphi=G R \varphi
$$

En multipliant (30) à droite par $\varphi^{\prime}$ et en intégrant, on en obtient une formulation variationnelle :

$$
\int_{\partial C}(1 / 2+H) \varphi \varphi^{\prime}=\int_{\partial C} G R \varphi \varphi^{\prime} \quad \forall \varphi^{\prime} .
$$

Maintenant, substituons l'expression (29) à $\varphi$ dans (32), et de même pour $\varphi^{\prime}$. On obtient une relation algébrique entre les approximations des différents opérateurs intervenant dans (31). Si, par abus de notation, on garde les mêmes lettres $G, H, R$ pour désigner ces approximations, cette relation s'écrit

$$
R=G^{-1}(1 / 2+H)
$$


d'où une méthode d'approximation de $R$. (Noter que la matrice $G$, qu'il faut inverser, est pleine, mais que sa dimension est le nombre de degrés de liberté portés par la frontière $\partial C$, soit beaucoup moins que le nombre total de degrés de liberté. Donc le caractère creux du système linéaire approchant (26) est bien préservé.) La matrice $R$ n'est pas symétrique (contrairement à l'opérateur $R$ qu'elle est censée approcher), donc on lui substitue sa partie symétrique $\left(R+R^{t}\right) / 2$. L'expérience numérique acquise jusqu'ici montre que cette méthode est satisfaisante, et un travail récent de Ren [14] donne des justifications concrètes et des exemples numériques. Par ailleurs, la convergence de cette méthode est prouvée [8]. On trouvera des résultats de calculs sur des problèmes réels dans [16].

\section{Conclusion de la première partie.}

Nous venons de décrire, en suivant le principe de la méthode de Galerkine, une méthode de calcul des courants de Foucault en trois dimensions, apte à traiter le cas de la figure 1 . On a mis en évidence l'utilité, comme guide, des formulations variationnelles, la possibilité, malgré le fait que le champ emplit tout l'espace, de restreindre le calcul à la zone conductrice, et l'intérêt, pour ce problème, d'éléments finis non conventionnels, liés aux arêtes du maillage.

\section{Deuxième partie}

\section{Exposé du problème.}

Cette partie est consacrée à l'extension de la méthode à des configurations analogues à celle de la figure 2. Dans celle-ci (imaginaire, mais typique), on a un cylindre conducteur $C_{1}$, ouvert en haut, un cylindre $C_{2}$ fermé à ses extrémités, et une plaque ferromagnétique $M_{1}$ enserrant $C_{1}$. Le tuyau $C_{1}$ comporte un défaut, ici une cavité, que l'on cherche à détecter par courants de Foucault. Pour cela on introduit dans $C_{1}$ une sonde, dont seul le noyau magnétique $M_{2}$ est représenté. Elle comporte aussi un bobinage enroulé autour de $M_{2}$, alimenté en courant alternatif à fréquence élevée $(100 \mathrm{kHz}$, par exemple). Les mesures de l'impédance de cette bobine dans différentes positions sur l'axe de $C_{1}$ renseignent sur la position et la taille du défaut. (Il y a en fait deux bobines identiques, montées en pont de Wheatstone, et c'est la différence de leurs impédances qui est mesurée.)

Cet exemple montre que la topologie des configurations rencontrées peut être assez complexe. Il peut y avoir plusieurs conducteurs et plusieurs corps magnétiques séparés, les conducteurs peuvent être creux, les cavités dans les conducteurs pourraient à leur tour contenir des parties magnétiques, etc.
Or un code de calcul doit pouvoir s'adapter à des situations inédites, donc, en principe, à toutes les configurations possibles. $\mathrm{Ce}$ point est souvent contesté, au nom du sens commun, sous le prétexte qu'il y a des limites à la complexité des configurations auxquelles un ingénieur (sous-entendu : quelqu'un de raisonnable...) peut vouloir s'intéresser. C'est oublier que le système logiciel n'est pas doué de sens commun, et qu'il faut lui donner la possibilité de distinguer les configurations légales, qu'il doit traiter correctement, des autres, résultant d'une erreur de description, ou d'une erreur accidentelle dans les données (voire de l'ambition excessive d'un usager déraisonnable). Autrement dit, il doit être construit conformément à des spécifications précises.

Une telle précision suppose un certain niveau de formalisme, qui fait appel au langage mathématique. Dans cet esprit, la classe de problèmes que nous souhaitons traiter peut se décrire ainsi (les clauses non détaillées sont les mêmes que dans la première partie) :

1) Sont données trois régions de l'espace, $S$, $C$ et $M$, bornées, disjointes, de frontières $\partial S$, วC, $\partial M$.

2) Dans $S, j^{s}$ est donné comme plus haut (point 2 de la section 1 , première partie).

3) Résistivité $\rho$ dans $C$, comme plus haut.

4) $C$ n'est pas connexe, mais chacune de ses parties est simplement connexe. De même pour $M$.

5) Perméabilité $\mu$ comme plus haut, sauf dans $M$, où on suppose $\mu=\mu_{0} / \varepsilon$, avec $\varepsilon$ très petit (et destiné, dans l'analyse mathématique, à tendre vers zéro).

6) La région $E-M$ est connexe.

Dans ces conditions, on veut calculer (à toutes fins ultérieures : représentations graphiques, calculs de forces, de pertes Joule, etc.) les courants induits dans $C$ et le champ magnétique partout.

La clause 4) permet d'avoir plusieurs conducteurs et plusieurs corps magnétiques, comme dans l'exemple de la figure 2. Mais elle exclut les cas où $C$ ou $M$ présentent des boucles (éventuellement entrelacées, comme sur la Fig. 3). On n'exclut pas, en revanche, que $C$ présente des cavités, qui peuvent à leur tour contenir d'autres corps magnétiques, ou d'autres conducteurs, etc.

(Le code Trifou n'est pas soumis à cette restriction. On la fait ici pour éviter de faire appel à des notions de topologie trop complexes. La théorie du cas général, nécessaire en particulier pour l'étude des transformateurs, fera l'objet d'un travail ultérieur.)

Sans la clause $6, M$ pourrait contenir des cavités (contenant des conducteurs, etc.). Mais à cause de l'effet d'écran des corps à haute perméabilité, les champs dans chaque cavité prise séparément sont 


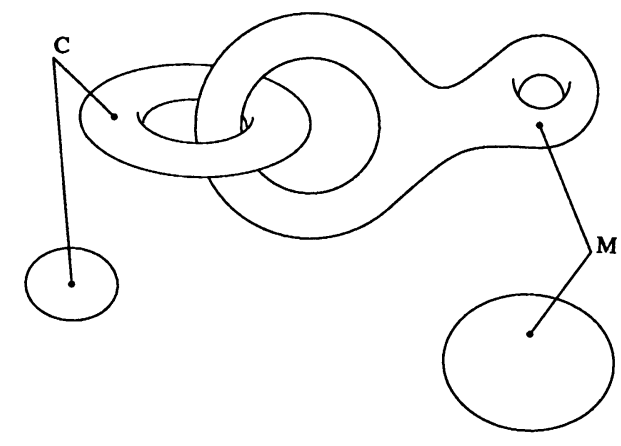

Fig. 3. - Une configuration imaginaire, non conforme aux spécifications décrites dans le texte.

[An ideal configuration which does not conform to specifications as given in the text.]

sans influence les uns sur les autres, donc peuvent être calculés indépendamment, de sorte que cette clause n'est pas une véritable restriction.

\section{Formulation.}

Commençons par les implications du fait que $\varepsilon$ est petit. Soit $h_{\varepsilon}$ le champ effectif pour $\varepsilon$ donné. D'après $(3,4)$, il est dans $H^{s}$ (défini en $(21)$ ) et vérifie

$$
\begin{aligned}
& i \omega \mu_{0}\left[\int_{E-M} h_{\varepsilon} \cdot h^{\prime}+\frac{1}{\varepsilon} \int_{M} h_{\varepsilon} \cdot h^{\prime}\right]+ \\
& \quad+\int_{C} \rho \operatorname{rot} h_{\varepsilon} \cdot \operatorname{rot} h^{\prime}=0 \quad \forall h^{\prime} \in H^{0} .
\end{aligned}
$$

Lorsque $\varepsilon$ tend vers zéro, on démontre (voir [4], [10], pour le mécanisme de la démonstration) ceci :

Théorème 1. - Lorsque $\varepsilon$ tend vers 0 , la solution $h_{\varepsilon} d e(34)$ converge (en moyenne quadratique, ainsi que son rotationnel) vers $h_{0}$ vérifiant les conditions

$$
\begin{array}{r}
h_{0} \in H^{s}, \quad h_{0}=0 \text { dans } M, \\
i \omega \mu_{0} \int_{E-M} h_{0} \cdot h^{\prime}+\int_{C} \rho \operatorname{rot} h_{0} \cdot \operatorname{rot} h^{\prime}=0 \\
\forall h^{\prime} \in H_{M}^{0},
\end{array}
$$

où $H_{M}^{0}$ est l'espace suivant

$$
H_{M}^{0}=\left\{h \in H^{0}: h=0 \text { dans } M\right\} .
$$

Ce résultat est conforme à l'intuition physique (le champ s'annule dans $M$, et la loi de Faraday continue d'être vérifiée, ce qu'exprime (36)). Il signifie que si $\varepsilon$ est assez petit, on peut substituer le modèle (35-36) au modèle initial (34). Assez petit est une notion relative, car des paramètres sans dimensions autre que $\varepsilon$ peuvent intervenir dans le problème. Il peut très bien arriver que $\varepsilon=10^{-1}$ soit petit dans telle situation alors que $\varepsilon=10^{-3}$ ne peut pas être tenu pour assez petit dans une autre. Nous ne poursuivons pas dans cette voie (qui mène à des problèmes de perturbations singulières).

Afin de simplifier les notations, on omettra désormais les indices $M$ et 0 . On redéfinit donc,- pour toute la suite :

$$
\begin{aligned}
& H=\left\{h \in \mathbb{L}_{\text {rot }}^{2}(E): h=0 \text { dans } M\right\}, \\
& H^{s}=\left\{h \in H: \operatorname{rot} h=j^{s} \text { dans } E-C\right\}, \\
& H^{0}=\{h \in H: \operatorname{rot} h=0 \text { dans } E-C\},
\end{aligned}
$$

et on appelle simplement $h$ le champ limite $h_{0}$, solution de (36). Il est dans $H^{s}$. C'est lui qu'on veut calculer, et il vérifie

$$
\begin{aligned}
i \omega \mu_{0} \int_{E-M} h \cdot h^{\prime}+\int_{C} \rho \operatorname{rot} h \cdot \operatorname{rot} h^{\prime} & =0 \\
\forall h^{\prime} & \in H^{0} .
\end{aligned}
$$

Il se présente la même difficulté que dans la première partie : $E-M$ est en général une région non bornée, qu'on ne peut pas mailler. On va donc se ramener à une discrétisation de la seule région conductrice, ainsi que de sa frontière, ainsi que (élément nouveau) de la frontière de $M$, grâce à l'opérateur de Poincaré-Steklov de la région $E-C-M$. Soit $\Omega$ cette région. On aura besoin des notations suivantes

$$
\begin{array}{ll}
M_{i} & : \text { une composante connexe de } \\
& M, i=1, \ldots, p ; \\
\partial M_{i} & : \text { la frontière de } M_{i} ; \\
C_{k} & : \text { une composante connexe de } C, k=1, \\
& \ldots, m ; \\
\partial_{l} C \quad: & \text { une composante connexe de } \partial C, l=1, \\
& \ldots, n ; \\
\Omega_{\alpha}, \partial \Omega_{\alpha}: \begin{array}{l}
\text { une composante connexe de } \Omega, \text { sa } \\
\text { frontière. }
\end{array} &
\end{array}
$$

Le nombre des $\Omega_{\alpha}$ est $n-m+1$. La figure 4 représente la situation générique.

On remarque d'abord que les composantes connexes des régions $\Omega$ et $E-C$ sont simplement connexes. Autrement dit, ces régions sont sans boucles. En effet, leurs complémentaires $C+M$ et $C$ ont cette propriété par hypothèse. Or, d'après un résultat général de topologie algébrique, deux régions complémentaires de l'espace ont le même nombre de boucles (ici, zéro).

Donc, tout champ irrotationnel dans $\Omega$ ou dans $E-C$ est un gradient.

Or $h$ vérifie, en tant qu'élément de $H^{s}$, rot $\left(h-h^{s}\right)=0$ dans $E-C$. Il existe donc un potentiel $\Phi$ tel que

$$
h=h^{s}+\operatorname{grad} \Phi \quad \text { dans } E-C .
$$

Comme $E-C$ a, comme $\Omega, n-m+1$ composantes connexes, et que $\Phi$ s'annule à l'infini, $\Phi$ est défini à 

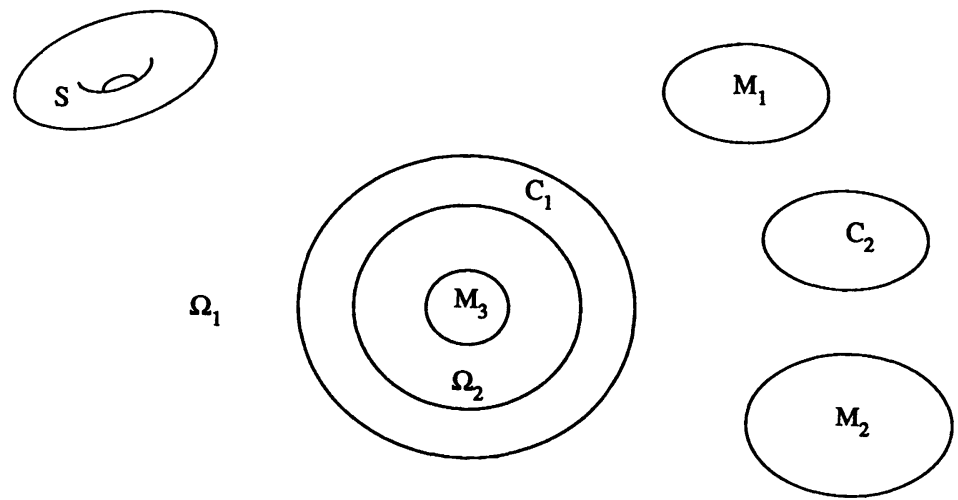

Fig. 4. - Ici, $M_{3}$ est intérieur à une cavité de $C_{1}$. On a $m=2, n=3, p=3$.

[Here, $M_{3}$ is enclosed by $C_{1}$, and $m=2, n=3, p=3$.]

$n-m$ constantes additives près. Pour les fixer, on impose la condition d'unicité suivante :

$$
\varphi \text { est de moyenne nulle sur la frontière }
$$

de chaque composante connexe bornée de $E-C$

où $\varphi$ est la restriction de $\Phi$ à $\partial C$. (On retrouvera une condition analogue plus loin, au niveau de la discrétisation.)

Reprenons la démarche familière: d'abord l'espace $H \Phi$, formé des couples $\{h, \varphi\}$ dont le premier est la restriction à $C$ d'un élément de $H$ (mais, cette fois, le $H$ défini en (37)) et le second une fonction sur $\partial \Omega$. Soit $h_{E}$ dans $H^{s}$, donc de la forme (40) dans $E-C$, soit $h$ sa restriction à $C, \varphi$ la restriction à $\partial \Omega$ du $\Phi$ de $(40):$ de nouveau $H \Phi^{s}$ est l'espace des couples $\{h, \varphi\}$ obtenus de cette façon. Enfin $H \Phi^{0}$ est celui des couples obtenus de même, mais lorsque $h_{E}$ parcourt $H^{0}$, au lieu de $H$. (Remarquer que cette fois $H \Phi^{s}$ et $H \Phi^{0}$ ne coïncident pas.)

L'opérateur de Poincaré-Steklov est cette fois celui de $\Omega=E-C-M$, défini ainsi. Soit $\varphi$ donnée sur $\partial \Omega$ et $\Phi$ vérifiant

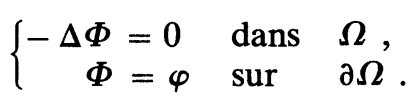

Alors $R \varphi=\partial \Phi / \partial n$, où $n$ est la normale sortant de $\Omega$. Le même calcul qu'en (25), mais cette fois dans $\Omega$, mène à la formulation variationnelle finale :

$$
\begin{aligned}
& i \omega \mu_{0}\left[\int_{C} h \cdot h^{\prime}+\int_{\partial C} n \cdot h^{s} \varphi^{\prime}+\right. \\
& \left.+\int_{\partial \Omega} R \varphi \varphi^{\prime}\right]+\int_{C} \rho \operatorname{rot} h \cdot \operatorname{rot} h^{\prime}=0 \\
& \forall\left\{h^{\prime}, \varphi^{\prime}\right\} \in H \Phi^{0}
\end{aligned}
$$

\section{Discrétisation.}

On va tenter de discrétiser comme dans la première partie, avec un degré de liberté par arête interne (dans $C$ ) et un par sommet frontalier $(\operatorname{sur} \partial \Omega$ ). Mais on va buter sur une difficulté : ces inconnues ne sont pas indépendantes.

Soit donc un maillage des conducteurs et des frontières des corps magnétiques. $\mathcal{A}$ et $\mathcal{A}_{0}$ désignent comme plus haut l'ensemble des arêtes et celui des arêtes internes, $\boldsymbol{S}$ celui de tous les sommets (ceux sur $\partial M$ inclus), $S_{0}$ celui des sommets intérieurs à $C$. On note $\mathcal{A}_{C}$ et $\mathcal{A}_{M}$ les arêtes dans $\partial C$ et $\partial M$. Soit $U$ l'espace des suites de nombres complexes de la forme

$$
u=\left\{u_{a}: a \in \mathcal{A}, u_{s}: s \in \mathcal{S}-\mathcal{S}_{0}\right\} .
$$

A tout $u$ on associe un élément de $H \Phi$, noté $\left\{h_{u}, \varphi_{u}\right\}$, par

$$
\begin{aligned}
& h=\sum_{a \in \mathcal{A}} u_{a} w_{a}, \\
& \varphi=\sum_{s \in \delta-\delta_{0}} u_{s} w_{s} .
\end{aligned}
$$

Pour faire pendant à $H \Phi^{s}$ et $H \Phi^{0}$, on définit $U^{s}$ et $U^{0}$ par

$$
\begin{aligned}
& U^{s}=\left\{u \in U:\left\{h_{u}, \varphi_{u}\right\} \in H \Phi^{s}\right\} \\
& U^{0}=\left\{u \in U:\left\{h_{u}, \varphi_{u}\right\} \in H \Phi^{0}\right\} .
\end{aligned}
$$

Les espaces $H \Phi_{\mathcal{C}}^{s}$ et $H \Phi_{\mathcal{C}}^{0}$ sont les images de $U^{s}$ et $U^{0}$ dans $H \Phi^{s}$ et $H \Phi^{0}$ par l'application (43) (44). Le problème discret, associé à (42) et au maillage $\mathcal{C}$ consistera donc à trouver $u \in U^{s}$ tel que

$$
\begin{gathered}
i \omega \mu_{0}\left[\int_{C} h_{u} \cdot h_{u}^{\prime}+\int_{\partial C} n \cdot h^{s} \varphi_{u^{\prime}}+\int_{\partial \Omega} R \varphi_{u} \varphi_{u^{\prime}}\right]+ \\
\quad+\int_{C} \rho \operatorname{rot} h_{u} \cdot \operatorname{rot} h_{u^{\prime}}=0 \quad \forall u^{\prime} \in U^{0}
\end{gathered}
$$


Toutefois, cette forme du problème n'est pas directement exploitable, car les composantes de $u$ (ou de $\left.u^{\prime}\right)$ ne sont pas indépendantes.

En effet, soit $u \in U^{s}$. La circulation de $h_{u}$ sur une arête $a$ de $\partial C$ (notée, rappelons-le,

$$
\int_{a} t \cdot h_{u}
$$

$t$ signifiant de façon générale le vecteur unitaire tangent à une courbe donnée) doit être égale à celle de $h^{s}+\operatorname{grad} \varphi_{u}$, par définition de $H \Phi^{s}$, de sorte que l'on a

$$
\int_{a} t \cdot h^{s}+u_{s}-u_{\sigma}=u_{a} \quad \forall a \in \mathcal{A}_{C} .
$$

De même

$$
\int_{a} t \cdot h^{s}+u_{s}-u_{\sigma}=u_{a}=0 \quad \forall a \in \mathcal{A}_{M} .
$$

De la même façon, si $u \in U^{0}$, on doit avoir

$$
\begin{array}{ll}
u_{a}=u_{s}-u_{\sigma} & \forall a \in \mathcal{A}_{C}, \\
u_{a}=u_{s}-u_{\sigma}=0 & \forall a \in \mathcal{A}_{M} .
\end{array}
$$

En fonction de tout cela, il faut sélectionner certaines variables (ou certaines combinaisons linéaires de variables) pour jouer le rôle de degrés de liberté. Nous attribuons un degré de liberté :

- à chaque arête du maillage interne à $C$,

- à chacun des $M_{i}, i=1, \ldots, p$,

- à chaque sommet de $\partial C$, sauf, pour chaque composante connexe bornée de $E-C$, un d'entre eux, pris sur la frontière de cette composante.

Cette dernière restriction est l'analogue discret de la condition (41). Soit $S *$ l'ensemble de ces sommets.

Soit $V$ l'ensemble des suites finies formées de ces degrés de liberté, soit

$$
v=\left\{v_{a}: a \in \mathcal{A}_{0}, v_{i}: i=1, \ldots, p, v_{s}: s \in S^{*}\right\} .
$$

Pour reconstituer un élément $u$ de $U^{0}$ à partir d'un élément $v$ de $V$, on procède ainsi :

$$
\begin{aligned}
& -u_{a}=v_{a} \text { pour tout } a \in \mathcal{A}_{0}, \\
& -u_{s}=v_{i} \text { pour tout sommet } s \text { de } \partial M_{i}, \\
& -u_{s}=v_{s} \text { pour } s \in \mathcal{S}^{*}, \\
& -u_{s}=0 \text { pour } s \in \mathcal{S}-\mathcal{S}_{0}-\mathcal{S}^{*},
\end{aligned}
$$

(on pourrait prendre une moyenne, par analogie avec la condition (41), mais ce serait une complication inutile, puisque les $u_{s}$ sont à une constante près de toute façon),

$$
\text { - } u_{a} \text { donné par (48) pour } a \in \mathcal{A}_{C} \cup \mathcal{A}_{M} \text {. }
$$

La reconstitution d'un élément de $u^{s}$ à partir d'un $v$ de $V$ est plus compliquée. On pose là aussi

$$
\begin{aligned}
& -u_{s}=v_{s} \text { pour } s \in \mathbf{S}^{*} \\
& -u_{s}=0 \text { pour } s \in \mathcal{S}-\boldsymbol{S}_{0}-\mathbf{S}^{*}
\end{aligned}
$$

puis

- $u_{s}=u_{i}$ pour $u n$ sommet de $\partial M_{i}$, appelons-le $s_{i}$,

- $u_{\sigma}$ donné par (47), de proche en proche, pour les autres sommets de $\partial M_{i}$,

- $u_{a}$ donné par (46) (47) pour $a \in \mathcal{A}_{C} \cup \mathcal{A}_{M}$.

Le calcul de la circulation de $h^{s}$ dans (46) et (47) est sans difficulté, puisque $h^{s}$ est connu de façon explicite par (19) et (20). La complication provient de ce qu'un sommet $\sigma$ de $\partial M_{i}$ peut être relié à $s_{i}$ par plusieurs chemins dans le graphe des arêtes. Il importe donc que la relation

$$
\sum_{i=1,2,3} \int_{a_{i}} t \cdot h_{s}=0
$$

pour les trois arêtes $a_{i}$ d'un même triangle, qui assure l'unité de la détermination de $u_{\sigma}$ par (47), soit préservée au cours du calcul. Du fait des erreurs d'arrondi, elle ne l'est jamais qu'approximativement. Une solution à ce problème technique consiste à sélectionner un ensemble minimal d'arêtes de $\mathcal{A}_{M}$ dont le graphe soit sans circuits, tout en visitant tous les sommets. (La détermination d'un tel arbre couvrant est un algorithme classique en théorie des graphes [11].)

On a enfin tous les éléments pour discrétiser (42). Soient $v$ et $v^{\prime}$ quelconques dans $V$. On leur associe $u \in U^{s}$ et $u^{\prime} \in U^{0}$ par les procédures qu'on vient de détailler. On pose alors

$$
\begin{aligned}
h & =\sum\left\{u_{a} w_{a}: a \in \mathcal{A}\right\}, \\
\varphi & =\sum\left\{u_{s} \lambda_{s}: s \in \mathcal{S}_{0}\right\},
\end{aligned}
$$

et de même pour $\left\{h^{\prime}, \varphi^{\prime}\right\}$ à partir de $u^{\prime}$, et on porte ces expressions dans (42). On obtient une forme quadratique par rapport aux $v$ et aux $v^{\prime}$, qui doit être nulle quel que soit $v^{\prime}$, ce qui donne un système linéaire par rapport aux $v$. C'était notre objectif.

\section{Conclusion de la deuxième partie.}

Comme la figure 2 le montre, il peut y avoir plusieurs conducteurs et plusieurs corps magnétiques dans une configuration réelle. La méthode décrite dans la première partie s'adapte à cette situation sans qu'il soit besoin de faire appel à des idées nouvelles, mais au prix de certaines précautions quant au choix des degrés de liberté : chaque corps magnétique ajoute un degré de liberté ou potentiel magnétique flottant, chaque cavité dans un conducteur en retranche un. Ces problèmes doivent retenir toute l'attention du programmeur.

\section{Conclusion.}

Nous avons présenté une méthode de résolution approchée du problème du calcul des champs magné- 
tiques et des courants de Foucault associés, en régime harmonique, dans une configuration tridimensionnelle générale, caractérisée par la présence de corps à grande perméabilité (mais excluant toutefois le cas de boucles conductrices et de boucles magnétiques entrelacées). L'expérience [16] a confirmé la validité de cette méthode dans des cas pratiques. L'étude met en évidence un certain nombre de difficultés d'implémentation, dues à des problèmes topologiques (conducteurs creux, cavités dans les corps magnétiques), et décrit en détail le choix des degrés de liberté et la procédure d'assemblage (dans le contexte de la méthode des éléments finis) pour faire face à ces difficultés.

\section{Remerciements.}

A P. Chaussecourte, F. Rioux-Damidau et J. C. Vérité.

\section{Bibliographie}

[1] Barton, M. L., Cendes, Z. J., New vector finite elements for three dimensional magnetic field computation, J. Appl. Phys. 61 (1987) 39193921.

[2] Bossavit, A., On the Numerical Analysis of Eddycurrent Problems Comp. Meth. Appl. Mech. Eng. 27 (1981) 303-318.

[3] Bossavit, A., On finite elements for the Electricity Equation in The Mathematics of Finite Elements and Applications IV, Ed. J. R. Whiteman (Acad. Press, London) 1982, pp. 85-92.

[4] Bossavit, A., Eddy-currents in a System of Moving Conductors in The Mechanical Behavior of Electromagnetic Solid Continua, Ed. G. A. Maugin (North-Holland, Amsterdam) 1984, pp. 345 350 .

[5] Bossavit, A., Two dual formations of the 3-D eddy currents problem, COMPEL 4 (1985) 103-106.

[6] Bossavit, A., On the condition « $h$ normal to the wall » in magnetic fields problems, Int. J. Numer. Meth. Eng. 24 (1987) 1541-1550.

[7] Bossavit, A., VÉRITÉ, J. C., The Trifou Code : Solving the 3-D Eddy-Currents Problem by Using $H$ as State Variable, IEEE Trans. Magn. 19 (1983) 2465-2470.

[8] Brezzi, F., Johnson, C. et Nédelec, J. C., On the Coupling of Boundary Integral and Finite Element Methods, Ecole Polytechnique, Rapport interne (Palaiseau) 1978.

[9] CARPENTER, J., Comparison of Alternative Formulations of 3-Dimensional Magnetic-Field and Eddy-Current Problems at Power Frequencies, Proc. IEEE 124 (1977) 1026-1034.
[10] Coulomb, J. L., Finite element three dimensional magnetic field computation, IEEE Trans. Magn. 165 (1981) 3241-3245.

[11] Gondran, M. et Minoux, M., Graphes et algorithmes (Eyrolles, Paris) 1979.

[12] NÉdElEC, J. C., Mixed finite elements in $\mathbb{R}^{3}$, Numer. Math. 35 (1980) 315-341.

[13] Pissanetzky, S., The Design of Superferric Magnets for the Superconducting Supercollider and the New Program Magnus for Three-Dimensional Magnetostatics, IEEE Trans. Magn. 216 (1985) 2457-2460.

[14] Ren, Z., Bouillault, F., Razek, A. et Vérité, J. C., Comparison of Different Boundary Integral Formulations when Coupled with Finite Elements in 3-D Electromagnetic Modrelling (soumis à IEEE Proc., Pt. A).

[15] Simkin, J. et Trowbridge, C. W., Three-Dimensional non-linear Electromagnetic Field Computation Using Scalar Potentials, IEEE Proc. $127 \mathrm{Pt}$. B (1980) 6.

[16] VÉRITÉ, J. C. et al., Trifou : un code de calcul tridimensionnel des courants de Foucault in Actes du Colloque MODELEC (La Grande Motte, 22-24 octobre 1984) Pluralis, Paris (1984) pp. 343-358.

[17] VÉRITÉ, J. C., Calculation of Multivalued Potentials in Exterior Regions IEEE Trans. Magn. 233 (1987) 1881-1887.

[18] Whitney, H., Geometric Integration Theory (Princeton U.P., Princeton) 1957.

[19] YosidA, K., Functional Analysis (Springer-Verlag, Berlin) 1965 